Article

\title{
Preparation and Physicochemical Properties of 10-Hydroxycamptothecin (HCPT) Nanoparticles by Supercritical Antisolvent (SAS) Process
}

Xiuhua Zhao, Yuangang Zu *, Ru Jiang, Ying Wang, Yong Li, Qingyong Li, Dongmei Zhao, Baishi Zu, Baoyou Zhang, Zhiqiang Sun and Xiaonan Zhang

Key Laboratory of Forest Plant Ecology, Northeast Forestry University, Ministry of Education, Harbin 150040, China; E-Mails: xiuhuazhao@nefu.edu.cn (X.Z.); 644677614@qq.com (R.J.); 41611005@qq.com (Y.W.); 724506183@qq.com (Y.L.); qingyong_li@163.com (Q.L.); 156828568@qq.com (D.Z.); zubaishi@163.com (B.Z.); zhangbaoyou@163.com (B.Z.); zhiqiangshun@163.com (Z.S.); 56573995@qq.com (X.Z.)

* Author to whom correspondence should be addressed; E-Mail: zygorl@ yahoo.com.cn; Tel.: +86-451-82191517; Fax: +86-451-82192082.

Received: 14 March 2011; in revised form: 13 April 2011 / Accepted: 18 April 2011 / Published: 20 April 2011

\begin{abstract}
The goal of the present work was to study the feasibility of 10-hydroxycamptothecin (HCPT) nanoparticle preparation using supercritical antisolvent (SAS) precipitation. The influences of various experimental factors on the mean particle size (MPS) of HCPT nanoparticles were investigated. The optimum micronization conditions are determined as follows: HCPT solution concentration $0.5 \mathrm{mg} / \mathrm{mL}$, the flow rate ratio of $\mathrm{CO}_{2}$ and $\mathrm{HCPT}$ solution 19.55, precipitation temperature $35{ }^{\circ} \mathrm{C}$ and precipitation pressure $20 \mathrm{MPa}$. Under the optimum conditions, HCPT nanoparticles with a MPS of $180 \pm 20.3 \mathrm{~nm}$ were obtained. Moreover, the HCPT nanoparticles obtained were characterized by Scanning electron microscopy, Dynamic light scattering, Fourier-transform infrared spectroscopy, High performance liquid chromatography-mass spectrometry, X-ray diffraction and Differential scanning calorimetry analyses. The physicochemical characterization results showed that the SAS process had not induced degradation of HCPT. Finally, the dissolution rates of HCPT nanoparticles were investigated and the results proved that there is a significant increase in dissolution rate compared to unprocessed HCPT.
\end{abstract}


Keywords: 10-hydroxycamptothecin; supercritical antisolvent; nanoparticles; preparation; physicochemical properties

\section{Introduction}

10-Hydroxycamptothecin (HCPT) (Figure 1), one of the natural camptothecin analogues, has been shown to have a broad spectrum of antitumor activity against breast, colon, lung, and ovarian cancers in clinical practice. More importantly, HCPT was shown to be more potent and less toxic than camptothecin(CPT) in experiments on animals and in human clinical evaluations, mainly in China [1,2]. However, due to its poor solubility in water or in physiologically acceptable organic solvents and in vitro and in vivo instability, clinical practical use of HCPT is limited [3]. The earlier clinical trials using its highly water-soluble sodium salt, $\mathrm{HCPT}-\mathrm{Na}^{+}$, which was the product of opening the lactone ring of HCPT, showed minimal anticancer activity and several unpredictable side effects such as myelosuppression, hemorrhagic cystitis, diarrhea, nausea, vomiting, and dermatitis. The lactone form is a more effective inhibitor of topoisomerase I compared to the carboxylate form, which has much lower anti-tumor activity [4]. Therefore, it is of great significance in developing the high performance delivery systems for the insoluble lactone form 10-HCPT. Recently, some novel delivery systems of HCPT have been reported, such as nanosuspensions [5], prodrugs [6-9], nanoparticles [10,11], microspheres [12-14], niosomes [3], emulsions [15] and polymeric micelle systems [16,17]. Among these tested approaches, nanosuspensions system, a sub-micro colloidal dispersion system, is considered as one of the most promising methods because of its characteristics in drug loading, release, delivery, stability and various options for administration, such as oral, parenteral, ocular and pulmonary pathways [18]. Production technologies of nanosuspensions currently used are pearl milling and high-pressure homogenization either in water or alternatively in mixtures of water with water miscible liquids or non-aqueous media, such as liquid polyethylene glycols or oils. Supercritical antisolvent (SAS) is a new micronization technology that has been developed in recent years. It is suitable to prepare nanocrystals of drugs or biologically active substances because of their low temperature and inertia. Until now, many bioactive materials have been successfully processed into nanocrystals [19-24].

However, no report has been carried out on the micronization of HCPT using SAS process. Therefore, the aims of this work are to study the feasibility of HCPT nanoparticle preparation by SAS process and to evaluate the SAS process factors which influence the mean particle size (MPS) of HCPT nanoparticles. The effects of concentration of HCPT solution, precipitation temperature, precipitation pressure and drug solution flow rate on the MPS of HCPT nanoparticles were studied by a four-factor, four-level OAD with an $\mathrm{OA}_{16}\left(4^{5}\right)$ matrix. Moreover, characterization of HCPT nanoparticles was analyzed by SEM, DLS, FTIR, LC-MS, XRD and DSC with the purpose of developing a suitable drug delivery system of cancer chemotherapy, nanosuspension of close-ring HCPT. 
Figure 1. Molecular structure of 10-hydroxycamptothecin (HCPT).

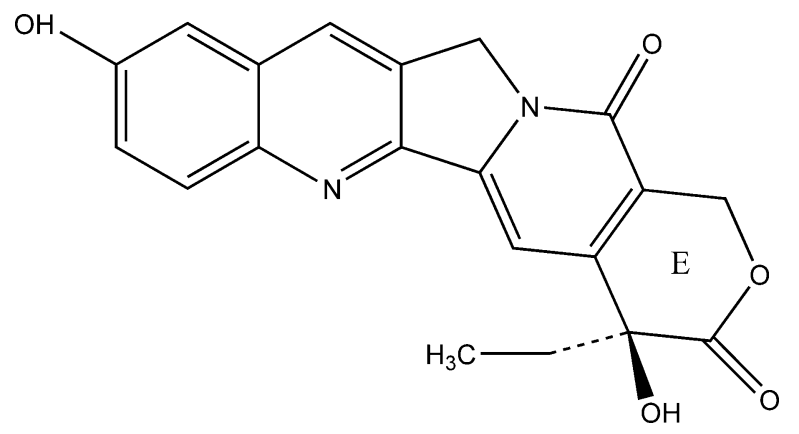

\section{Experimental Section}

\subsection{Materials}

10-HCPT (98.5\% purity) was obtained from Zhejiang Hisun Pharmaceutical Co. Ltd (PR China). High purity $\mathrm{CO}_{2}$ (99.99\% purity) was purchased from Liming Gas Company of Harbin (PR China). Dimethyl sulfoxide (DMSO, 98.5\% purity) was purchased from Sigma Aldrich. All solutions used in HPLC analysis were of HPLC grade and filtered using a $0.22 \mu \mathrm{m}$ membrane filter (YDHITECH, China) with a filtration system (SHB-III, China).

\subsection{Apparatus and Supercritical Anti-Solvent (SAS) Process}

The schematic of SAS process is shown in Figure 2. The apparatus consists of a precipitation chamber and a gas-liquid separation chamber. The $\mathrm{CO}_{2}$ is cooled with a cooler before being compressed by a liquid pump and the pressure is controlled by a back pressure regulator. Afterwards, the $\mathrm{CO}_{2}$ is pre-heated in a heat exchanger and enters into the precipitation chamber. Simultaneously, the solution is pumped, heated and fed to the $1000 \mathrm{~mL}$ precipitation chamber through a stainless steel nozzle. This nozzle is located in a distinct inlet point from the $\mathrm{CO}_{2}$, but also in the top of the precipitation chamber. A stainless steel frit vessel of $200 \mathrm{~nm}$ was put into the precipitation chamber to collect the micronized particles and to let the $\mathrm{SC}-\mathrm{CO}_{2}$ /organic solvent mixture pass through. The flow rate of the mixture that leaves the precipitator is controlled by a valve located between the precipitation chamber and the gas-liquid separation chamber. Here the mixture undergoes a decompression (pressure $<5 \mathrm{MPa}$ ) to induce the separation of the $\mathrm{CO}_{2}$ from the organic solvent. Further information and a schematic representation of the apparatus have been given elsewhere [25].

An SAS experiment begins by delivering supercritical $\mathrm{CO}_{2}$ to the precipitation chamber until the desired pressure is reached. $\mathrm{CO}_{2}$ steady flow of $8.5 \mathrm{~kg} / \mathrm{h}$ is established; then pure DMSO is sent through the liquid pump to the precipitation chamber with the aim of obtaining steady state composition conditions during the HCPT precipitation. At this point, the flow of the pure DMSO is stopped and the HCPT/DMSO liquid solution is delivered through the nozzle of $150 \mu \mathrm{m}$. Once injected the fixed quantity of HCPT/DMSO solution, the liquid pump is stopped. However, supercritical $\mathrm{CO}_{2}$ continues to flow for $30 \mathrm{~min}$ to wash the stainless steel frit vessel of $200 \mathrm{~nm}$ from the residual content of liquid solubilized in the supercritical antisolvent. If the final purge with pure $\mathrm{CO}_{2}$ is not performed, DMSO condenses during the depressurization and redissolves the micronized HCPT. 


\subsection{Optimization of SAS Process}

An orthogonal $\mathrm{OA}_{16}(4)^{5}$ test design was used to investigate the optimal micronized condition of HCPT. The SAS experiment was carried out with 4 factors and 4 levels, namely: concentration of HCPT solution $(0.5,1.5,3.0,5.0 \mathrm{mg} / \mathrm{mL})$, drug solution flow rate $(3.3,6.6,9.9,13.2 \mathrm{~mL} / \mathrm{min})$, precipitation temperature $\left(35,46,57,68^{\circ} \mathrm{C}\right)$ and precipitation pressure $(10,15,20,25 \mathrm{MPa})$. The range of each factor level was based on the results of preliminary experiments. The mean particle size (MPS) of micronized HCPT (nm) was the dependent variable. The micronized HCPT obtained from the above 16 tests (Table 1) was operated following the method in the Section 3.2.

Figure 2. Schematic illustration of the supercritical antisolvent (SAS) experimental apparatus.

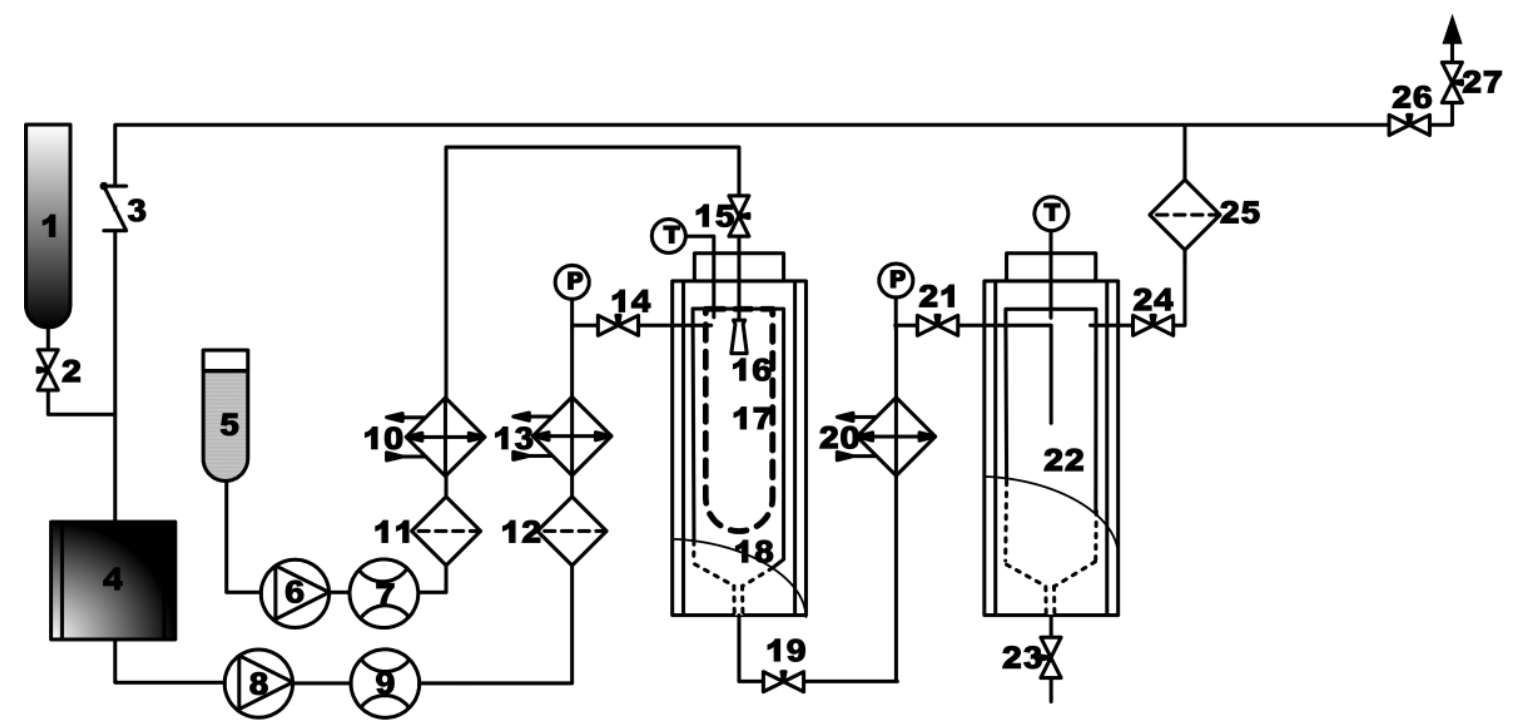

1. $\mathrm{CO}_{2}$ cylinder; 2. 14, 15, 19, 21, 23, 24, 26 and 27. Valves; 3. Check valve; 4. $\mathrm{CO}_{2}$ cooler; 5. Liquid solution supply; 6. Liquid pump; 7 and 9. Flow meter; 8. $\mathrm{CO}_{2}$ pump; 10, 13 and 20. Heat exchangers; 11, 12 and 25. Filters; 16. Nozzle; 17. Stainless steel frit vessel of $200 \mathrm{~nm} ; 18$. Precipitation chamber; 22. Gas-liquid sepatation chamber.

\subsection{Physicochemical Properties of HCPT Nanoparticles}

\subsubsection{Research of the Morphology}

The morphology analysis of HCPT particles was carried out using SEM (Quanta 200, FEI). The samples were prepared by direct deposition of the powders onto a carbon tape placed on the surface of an aluminium stub. Before analysis, the samples were coated with gold for 4 min using a sputter coater.

\subsubsection{Dynamic Light Scattering (DLS)}

Micronized HCPT was suspended in filtered pure water and special cares were taken to eliminate dust and to avoid the aggregation of particles. The water was pre-saturated with HCPT to avoid dissolution of the micronized particles. The suspension was analyzed in DLS (ZetaPALS, Brookhaven Instruments). Every measurement was repeated at least three times. 


\subsubsection{FTIR Analysis}

The HCPT particles were diluted with $\mathrm{KBr}$ mixing powder at $1 \%$ and separately pressed to obtain self-supporting disks. The FTIR spectrum was obtained by IRAffinity-1 (SHIMADZU, Jepan) and recorded in the wave number range of $4000-500 \mathrm{~cm}^{-1}$ at a resolution of $4 \mathrm{~cm}^{-1}$.

\subsubsection{LC-MS Analysis}

The HCPT particles were dissolved in ethanol and LC-MS was obtained by analyst 1.4 of AB API 3000 (USA). The mass spectrometer was operated in positive ion mode.

\subsubsection{XRD Analysis}

$10 \mathrm{mg}$ samples of HCPT particles, forming a weighted dispersion on a glass slide, were evaluated using an X-ray powder diffractometer (Philips, Xpert-Pro, The Netherlands). The samples were irradiated using a $\mathrm{Cu}$ target tube at $30 \mathrm{~mA}$ and $50 \mathrm{kV}$. The samples were filled to the same depth inside the sample holder by leveling with a spatula. The scanning rate $\left(5^{\circ} \mathrm{min}\right.$.) was constant for all XRD analysis.

\subsubsection{DSC Analysis}

Thermal analysis was carried out using DSC (TA instruments, model DSC 204) for HCPT particles. All thermal analyses were performed in an inert atmosphere $\left(\mathrm{N}_{2}\right)$. Analysis was performed for $5.0 \mathrm{mg}$ samples at a temperature heating rate of $5{ }^{\circ} \mathrm{C} / \mathrm{min}$ and a temperature range of $20-265{ }^{\circ} \mathrm{C}$.

\subsubsection{Dissolution Studies in Vitro}

$100 \mathrm{mg}$ sample was added to a $500 \mathrm{~mL}$ dissolution medium $\left(6.4 \mathrm{~g} \mathrm{Na}_{2} \mathrm{HPO}_{4}, 12 \mathrm{H} 2 \mathrm{O} ; 0.6 \mathrm{~g}\right.$ $\mathrm{KH}_{2} \mathrm{PO}_{4} ; 5.85 \mathrm{~g} \mathrm{NaCl}$ in $1000 \mathrm{~mL}$ distilled water; $\mathrm{pH}$ 7.4). Bath temperature and paddle speed were set at $37 \pm 0.5{ }^{\circ} \mathrm{C}$ and $100 \mathrm{rpm}$. At selected periods of 5, 10, 15, 30, 45, 60 and $120 \mathrm{~min}, 2 \mathrm{~mL}$ aliquots were withdrawn without media replacement, filtered, and analyzed using reversed-phase HPLC (waters) at $\lambda=266 \mathrm{~nm}$. The data was expressed as a mean value \pm S.D $(\mathrm{n}=6)$. The percentage of cumulated dissolution was defined as the sum of the mass of dissolved HCPT at time $t$, divided by the mass of added HCPT (100 mg). The dissolution profiles were plotted as the percentage of cumulated dissolution versus incubation time.

Filtered samples were appropriately diluted with methanol and assayed for HCPT concentration by HPLC. Chromatographic analyses were performed on a waters HPLC system consisting of a pump (Model 1525), an auto-sampler (Model 717 plus), UV detector (Waters 2487 Dual $\lambda$ Absorbance Detector). The $\mathrm{C}_{18}$ reverse phase column (Diamonsil, $\mu \mathrm{m}, 4.6 \mathrm{~mm} \times 250 \mathrm{~mm}$, Dikma Technologies) was used at room temperature. The mobile phase consisted of $70 \%$ acetonitrile delivered at $1.0 \mathrm{~mL} / \mathrm{min}$. The injection volume was $10 \mu \mathrm{L}$. The signal was monitored at $266 \mathrm{~nm}$. 


\section{Results and Discussion}

\subsection{Optimization Study}

The first step in the SAS micronization process is to optimize the operating conditions to obtain a minimum MPS of micronized HCPT. Since various parameters potentially affect the micronization process, the optimization of the experimental conditions is a critical step in developing an SAS method. In fact, the concentration of drug solution, precipitation temperature, precipitation pressure and drug solution flow rate are generally considered the most important factors that affect the MPS of HCPT. The investigated levels of each factor were selected depending on the preliminary experiment results of the single-factor. The optimization of the suitable operating conditions in SAS can be carried out step by step or by using an experimental design. In the present study, all selected factors were examined using an orthogonal $\mathrm{OA}_{16}\left(4^{5}\right)$ test design. The total evaluation index was used to analyse by statistical methods. The analysis results of orthogonal test, performed by statistical software Design Expert 7.0, are presented in Table 1. Table 2 lists the data of the analysis of variance (ANOVA) table of this experiment. The results of experiments presented in Table 2 indicate that the MPS of micronization HCPT varied between 210.5 and $900.7 \mathrm{~nm}$. The maximum MPS was found for trial 16 and the lowest for trial 2. The factors influence the MPS of micronization HCPT were listed in a decreasing order as follows: $\mathrm{A}>\mathrm{C}>\mathrm{B}>\mathrm{D}$ according to the $R$ value. So, the minimum MPS of micronization HCPT was obtained when the concentration of HCPT solution, precipitation temperature, precipitation pressure and drug solution flow rate were $\mathrm{A}_{1} \mathrm{~B}_{1} \mathrm{C}_{3} \mathrm{D}_{2}\left(0.5 \mathrm{mg} / \mathrm{mL}, 35{ }^{\circ} \mathrm{C}\right.$, $20 \mathrm{MPa}$ and $6.6 \mathrm{~mL} / \mathrm{min}$ ), respectively. Through a confirmatory test, smaller micronized HCPT was obtained, with a MPS of $180 \pm 20.3 \mathrm{~nm}$.

Table 1. Orthogonal array design matrix $\mathrm{L}_{16}(4)^{5}$ and experimental results.

\begin{tabular}{ccccccc}
\hline Trial No. & $\mathbf{A}$ & $\mathbf{B}$ & $\mathbf{C}$ & $\mathbf{D}$ & Error & $\begin{array}{c}\text { MPS (nm) } \pm \text { SD } \\
(\mathbf{n}=\mathbf{3})\end{array}$ \\
\hline 1 & $1(0.5)$ & $1(35)$ & $1(10)$ & $1(3.3)$ & 1 & 381.2 \\
2 & $1(0.5)$ & $2(46)$ & $2(15)$ & $2(6.6)$ & 2 & 210.5 \\
3 & $1(0.5)$ & $3(57)$ & $3(20)$ & $3(9.9)$ & 3 & 350.0 \\
4 & $1(0.5)$ & $4(68)$ & $4(25)$ & $4(13.2)$ & 4 & 424.8 \\
5 & $2(1.5)$ & $1(35)$ & $2(15)$ & $3(9.9)$ & 4 & 409.4 \\
6 & $2(1.5)$ & $2(46)$ & $1(10)$ & $4(13.2)$ & 3 & 458.3 \\
7 & $2(1.5)$ & $3(57)$ & $4(25)$ & $1(3.3)$ & 2 & 437.9 \\
8 & $2(1.5)$ & $4(68)$ & $3(20)$ & $2(6.6)$ & 1 & 446.0 \\
9 & $3(3.0)$ & $1(35)$ & $3(20)$ & $4(13.2)$ & 2 & 350.6 \\
10 & $3(3.0)$ & $2(46)$ & $4(25)$ & $3(9.9)$ & 1 & 428.2 \\
11 & $3(3.0)$ & $3(57)$ & $1(10)$ & $2(6.6)$ & 4 & 752.8 \\
12 & $3(3.0)$ & $4(68)$ & $2(15)$ & $1(3.3)$ & 3 & 641.1 \\
13 & $4(5.0)$ & $1(35)$ & $4(25)$ & $2(6.6)$ & 3 & 402.9 \\
14 & $4(5.0)$ & $2(46)$ & $3(20)$ & $1(3.3)$ & 4 & 474.5 \\
15 & $4(5.0)$ & $3(57)$ & $2(15)$ & $4(13.2)$ & 1 & 720.6 \\
16 & $4(5.0)$ & $4(68)$ & $1(10)$ & $3(9.9)$ & 2 & 900.7 \\
\hline
\end{tabular}


Table 1. Cont.

\begin{tabular}{ccccccc}
\hline Trial No. & A & B & C & D & Error & $\begin{array}{c}\text { MPS (nm) } \pm \text { SD } \\
(\mathbf{n}=\mathbf{3})\end{array}$ \\
\hline$K_{1}{ }^{a}$ & 341.6 & 386.0 & 623.3 & 483.7 & 494.0 & \\
$K_{2}$ & 437.9 & 392.9 & 495.4 & 453.1 & 475.0 & \\
$K_{3}$ & 543.2 & 565.3 & 405.3 & 522.1 & 463.1 & \\
$K_{4}$ & 624.7 & 603.2 & 423.5 & 488.6 & 515.4 & \\
$R^{b}$ & 283.1 & 217.1 & 218.0 & 69.0 & 52.3 & \\
$\begin{array}{c}\text { Optimal } \\
\text { level }\end{array}$ & $\mathrm{A}_{1}$ & $\mathrm{~B}_{1}$ & $\mathrm{C}_{3}$ & $\mathrm{D}_{2}$ & & \\
\hline
\end{tabular}

A, Concentration of HCPT solution $(\mathrm{mg} / \mathrm{mL}) ; \mathrm{B}$, Precipitation temperature $\left({ }^{\circ} \mathrm{C}\right)$; C, Precipitation pressure (MPa); D, Drug solution flow rate $(\mathrm{mL} / \mathrm{min})$

$a K_{i}=\sum$ (mean particle size at $\left.\mathrm{A}_{\mathrm{i}}\right) / 4$, the mean values of MPS for a certain factor at each level with standard deviation.

$b R=\max K_{i}-\min K_{i}$.

Table 2. ANONA analysis of four parameters for SAS micronization of 10-hydroxycamptothecin (HCPT).

\begin{tabular}{lccccc}
\hline \multicolumn{1}{c}{ Source } & $\begin{array}{c}\text { Sum of } \\
\text { squares } \\
(\mathbf{S S})\end{array}$ & $\begin{array}{c}\text { Degrees of } \\
\text { freedom } \\
(\mathbf{d f})\end{array}$ & $\boldsymbol{F}$-ratio & $\boldsymbol{F}_{\boldsymbol{0 . 0 5}}$ & $\begin{array}{c}\text { Type of } \\
\text { effect }\end{array}$ \\
\hline (A) Concentration of HCPT solution & 182618.6 & 3 & 29.038 & 9.28 & Significant \\
(B) Precipitation temperature & 154724 & 3 & 24.602 & 9.28 & Significant \\
(C) Precipitation pressure & 117408.4 & 3 & 18.669 & 9.28 & Significant \\
(D) Drug solution flow rate & 9585.187 & 3 & 1.524 & 9.28 & \\
Error & 6289.02 & 3 & & & \\
\hline
\end{tabular}

\subsection{Effect of Operating Conditions on the MPS of Micronized HCPT}

Analysis of variance showed that the concentration of HCPT solution, precipitation temperature and precipitation pressure had a significant effect on the MPS of micronized HCPT $(p<0.05)$ in the selected ranges (Table 2). However, drug solution flow rate has no significant influence on the MPS.

An orthogonal experimental design allows the separation of the effects of each individual factor investigated. However, it is interesting to analyze the effects of two factors on MPS. A nonlinear regression was applied using Design Expert 7.0 software to plot surface responses. The correlation related the MPS of micronized HCPT to the main variables-concentration of HCPT solution, precipitation temperature and precipitation pressure.

Equation (1) is expressed as follows:

$$
\begin{aligned}
& Y=915.8459+191.1776 \times A-8.0994 \times B-67.5255 \times C+0.3836 \times A \times B-4.6189 \times A \times C \\
& +0.3836 \times A \times B-4.6189 \times A \times C+0.2973 \times B \times C-10.4852 \times A^{2}+0.0640 \times B^{2}+1.4603 \times C^{2}
\end{aligned}
$$

Where $Y$ is the MPS of micronized HCPT (nm), $A$ is the concentration of HCPT solution (MPa), $B$ is the precipitation temperature $\left({ }^{\circ} \mathrm{C}\right)$, and $C$ is precipitation pressure $(\mathrm{MPa})$. The correlation coefficient is 0.9591 . 
Figure 3 shows the three-dimensional response surfaces which were constructed to show the effects of the SAS process variables on MPS of micronized HCPT $(Y)$. Figure 3a-c shows the interaction effect of precipitation temperature and concentration of HCPT solution, precipitation pressure and concentration of HCPT solution, and precipitation pressure and precipitation temperature on the MPS, respectively. The MPS of micronized HCPT was found to increase with increasing precipitation temperature and concentration of HCPT solution and decrease with increasing precipitation pressure.

Figure 3. Response surface for MPS of micronized HCPT obtained by nonlinear regression.
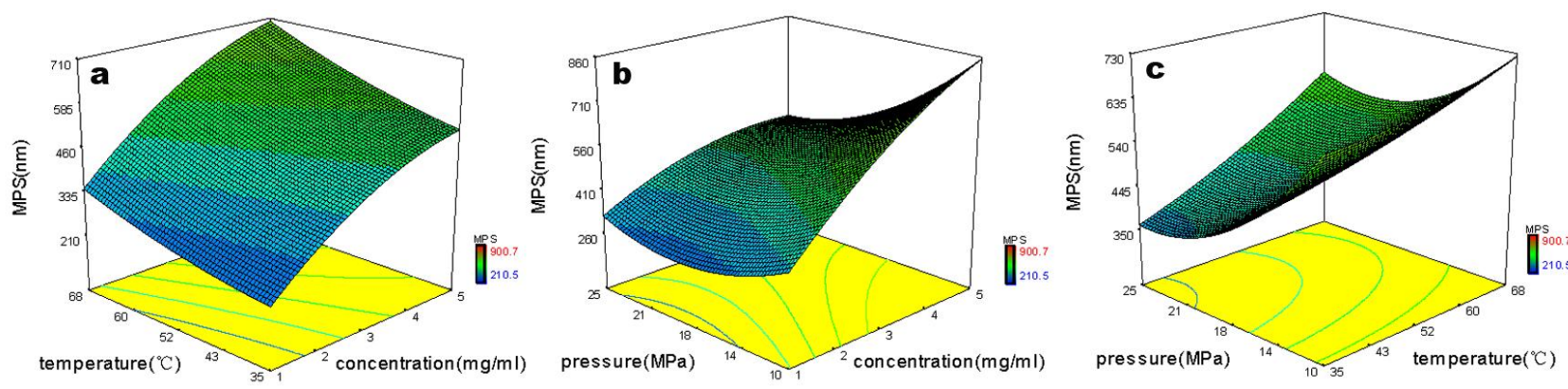

\subsection{Morphology of Processed HCPT}

Figure 4 shows the SEM photomicrograph of original HCPT particles. As we can see, commercial HCPT particles are not uniform and basically pillar or bar shaped. The average particle size was found to be about $1 \times 1 \times 50 \mu \mathrm{m}$. Figure 5a shows the SEM images of HCPT nanoparticles precipitated from DMSO under the optimum SAS process condition $(0.5 \mathrm{mg} / \mathrm{mL}$ of concentration of HCPT solution, $35{ }^{\circ} \mathrm{C}$ of precipitation temperature, $20 \mathrm{MPa}$ of precipitation pressure and $6.6 \mathrm{~mL} / \mathrm{min}$ of HCPT solution flow rate), from which it is obvious that the HCPT nanoparticles are close to ellipsoidal with the MPS of $180 \pm 20.3 \mathrm{~nm}$ and the particle size distribution (PSD) is shown in Figure $5 \mathrm{~b}$.

Figure 4. Scanning electron microscope (SEM) image of unprocessed HCPT.

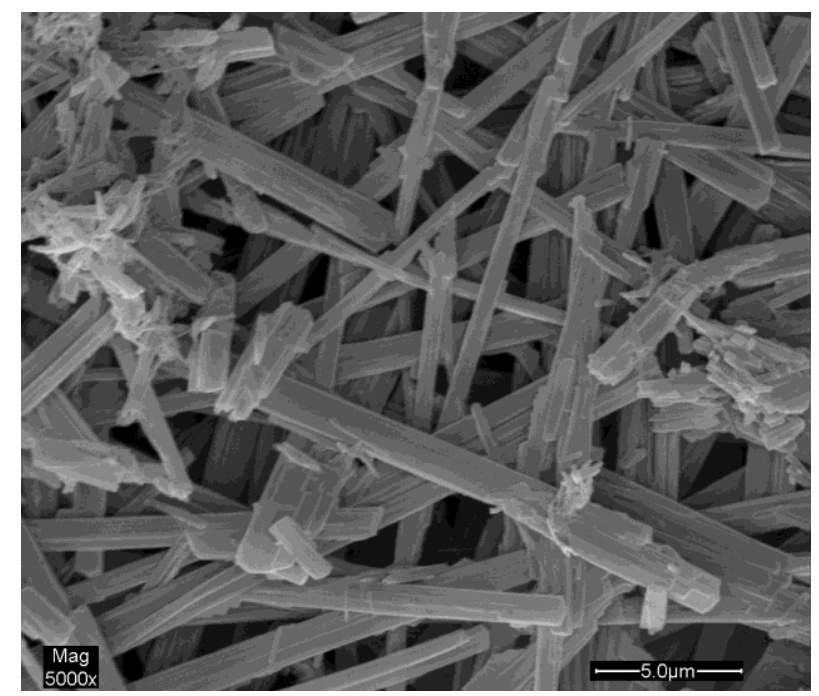


Figure 5. SEM image (a) and particle size distribution (PSD) (b) of HCPT nanoparticles.
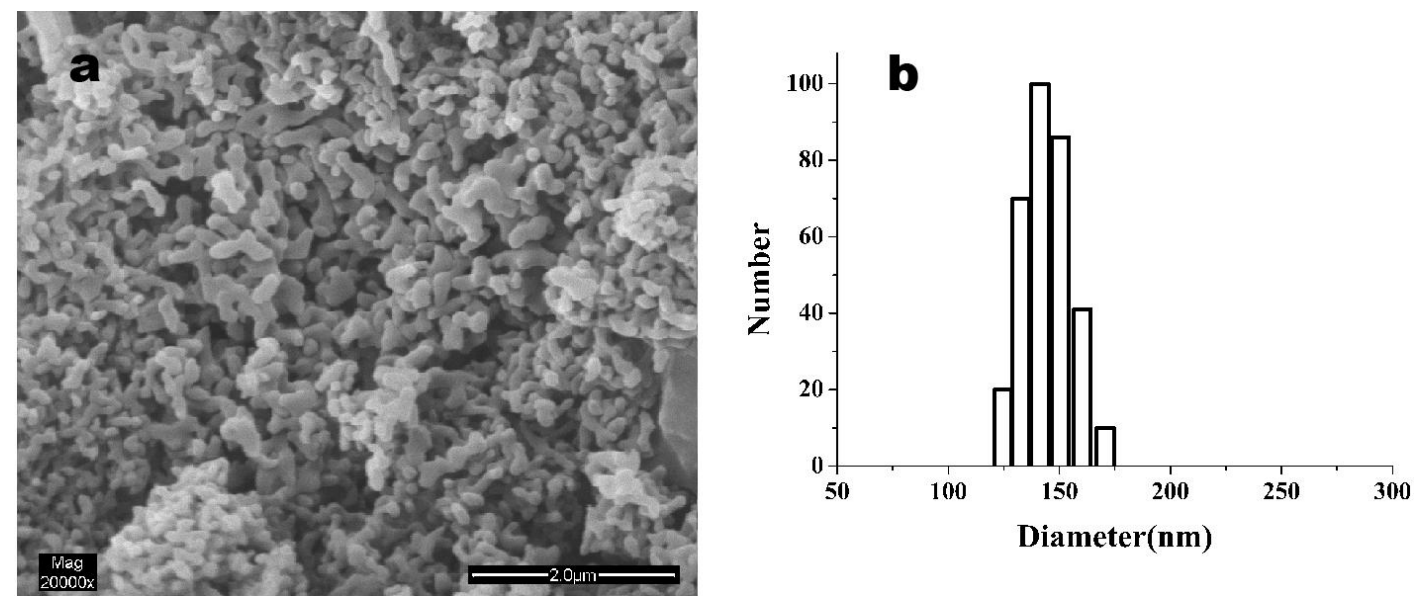

\subsection{Physicochemical Properties of HCPT Nanoparticles}

\subsubsection{FTIR Analysis}

We performed some analysis on original HCPT particles and HCPT nanoparticles to obtain information on the change of chemical structure after SAS processing. It can be seen that FTIR spectra between unprocessed and HCPT nanoparticles do not show any significant differences (Figure 6). Several characteristic IR absorption bands and their assignments are shown as follows: 3494 and $3346 \mathrm{~cm}^{-1}$ (the $\mathrm{O}-\mathrm{H}$ stretching mode), $3100-2750 \mathrm{~cm}^{-1}$ (the $\mathrm{C}-\mathrm{H}$ stretching vibration), $1723 \mathrm{~cm}^{-1}$ ( $\mathrm{C}=\mathrm{O}$ stretching vibration of lactone), $1653 \mathrm{~cm}^{-1}$ (the $\mathrm{C}=\mathrm{O}$ stretching vibration of amide), 1593 and $1502 \mathrm{~cm}^{-1}$ (the aromatic ring mode), 1266 and $1051 \mathrm{~cm}^{-1}$ (the $\mathrm{C}-\mathrm{O}$ and/or $\mathrm{C}-\mathrm{N}$ stretching mode).

Figure 6. FTIR spectra of unprocessed HCPTand HCPT nanoparticles.

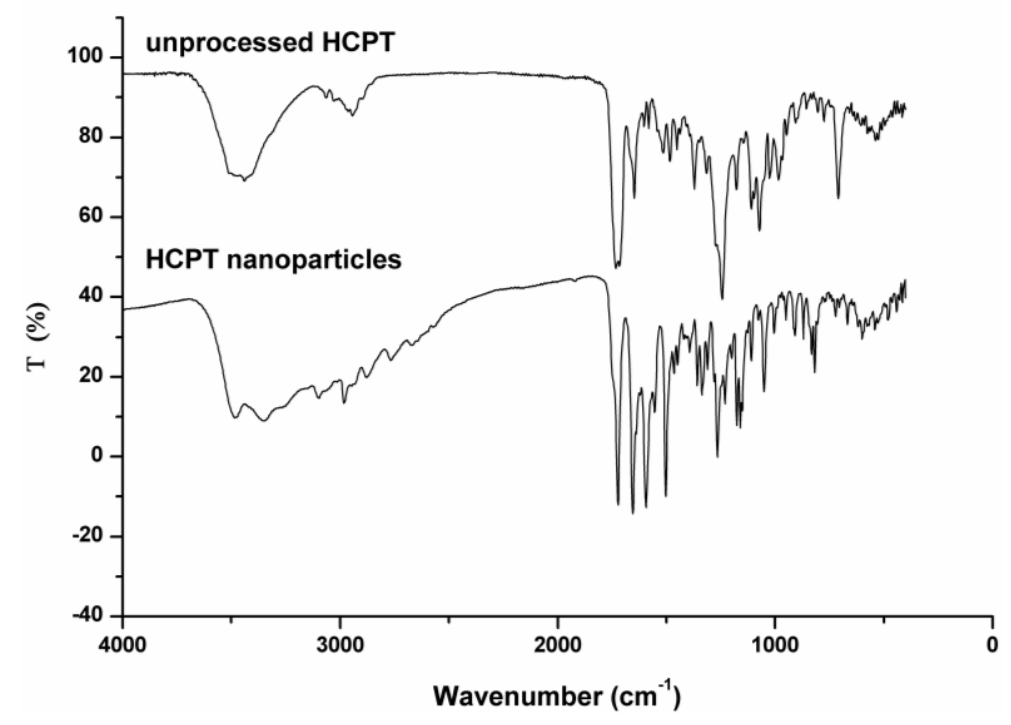

\subsubsection{LC-MS Analysis}

The unprocessed and processed HCPT samples were evaluated by using LC-MS to determine the molecular weights, as shown in Figure 7. It can be seen that no modification occurred in molecular 
weight (364.5). The two forms exhibit the mass spectral peak, at 365.5, coming from the $\left[\mathrm{C}_{20} \mathrm{H}_{16} \mathrm{~N}_{2} \mathrm{O}_{5}+\mathrm{H}\right]^{+}$peak. This explains why there were no varieties about chemistry structure of HCPT before/after SAS process. Therefore, the SAS process has not induced degradation of HCPT.

Figure 7. LC-MS spectra of the unprocessed HCPT (a) and HCPT nanoparticles (b).
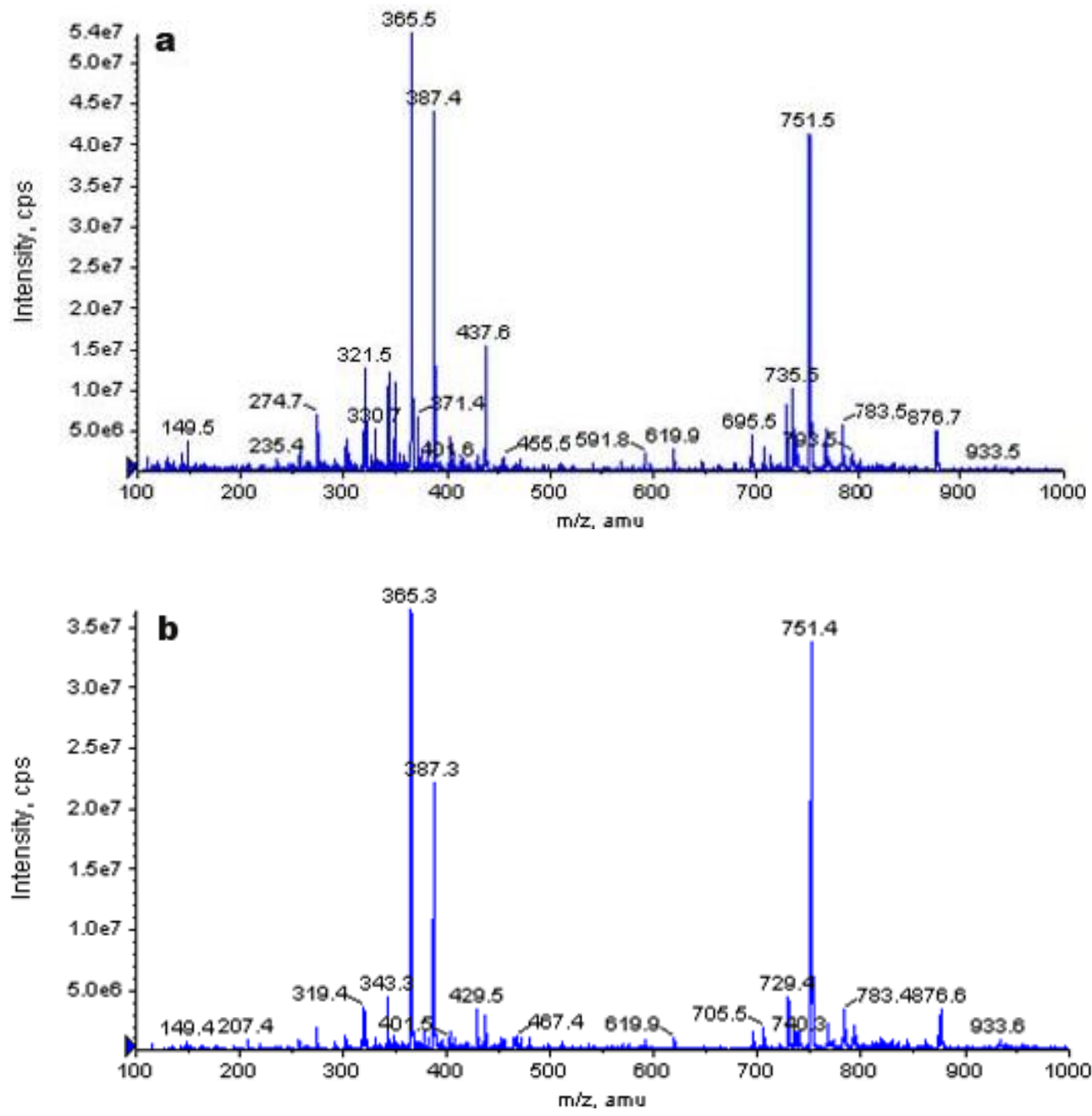

\subsubsection{X-ray Analysis}

In order to further investigate particles, crystalline XRD analysis was performed. Figure 8 shows the XRD results for unprocessed HCPT particles and HCPT nanoparticles. The presence of several distinct peaks in the XRD of unprocessed HCPT at the diffraction angles of $2 \theta=13.821^{\circ}, 11.659^{\circ}$, $25.640^{\circ}, 13.521^{\circ}, 6.900^{\circ}, 28.260^{\circ}, 27.919^{\circ}$ and $27.219^{\circ}$ reveals that the drug is present as a crystalline form. On the other hand, HCPT nanoparticles were characterized by the complete absence of any diffraction peak corresponding to unprocessed HCPT. This fact suggests that HCPT particles after SAS processing are no longer present in crystalline form, but exist in the amorphous state. Less crystalline smaller drug particles are higher in the dissolution rate or bioavailability than crystals and, thus, the therapeutic action is obtained in shorter times. 
Figure 8. XRD patterns of the unprocessed HCPT and HCPT nanoparticles.

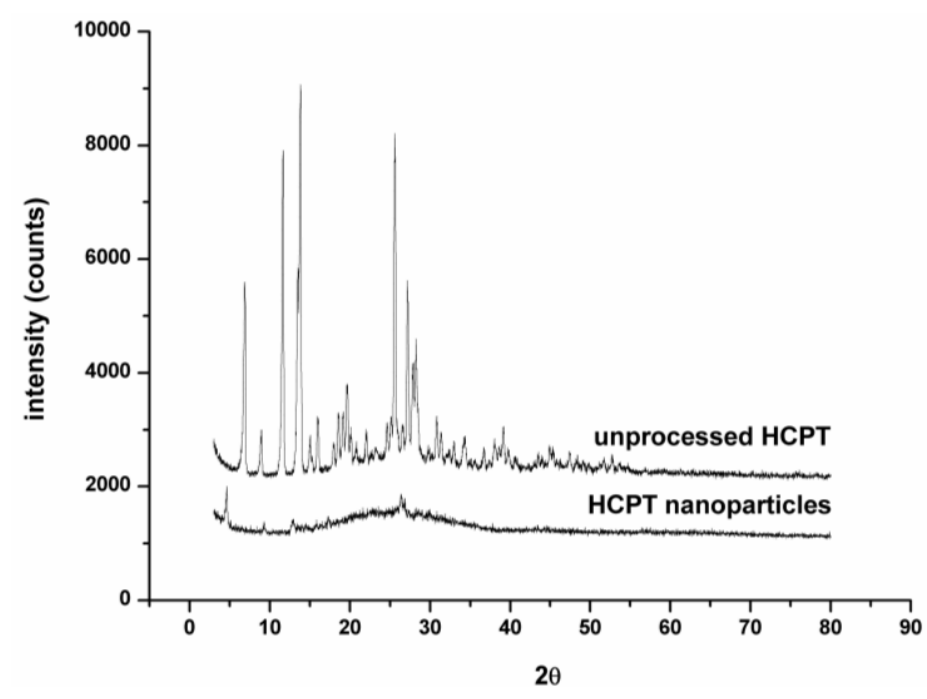

\subsubsection{DSC Analysis}

Figure 9 shows the DSC curves of unprocessed HCPT particles and HCPT nanopaticles. The DSC curves of unprocessed HCPT, a sharp endotherm at $279.93{ }^{\circ} \mathrm{C}$ were observed. The sharp endotherm at $279.93{ }^{\circ} \mathrm{C}$ might be due to the melting point of HCPT. However, no endotherms were observed in the DSC curves for HCPT nanoparticles. This result indicates that HCPT is no longer present as a crystalline form when processed using SAS, but exists in the amorphous state.

Figure 9. DSC results of the unprocessed HCPT and HCPT nanoparticles.

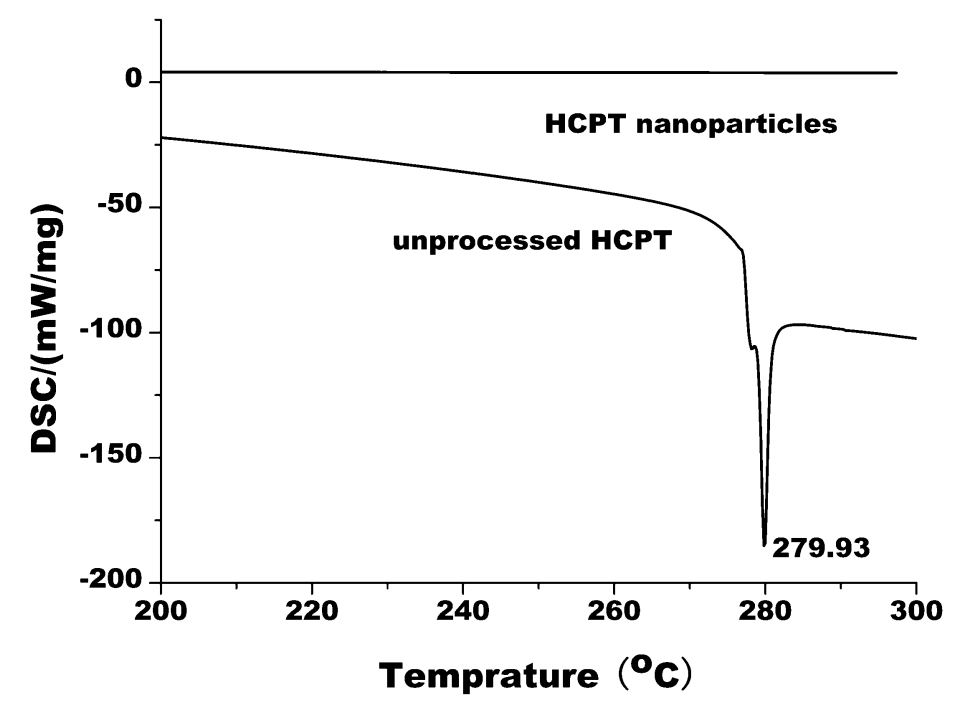

\subsubsection{Dissolution Studies in Vitro}

Dissolution profiles of HCPT particles are shown in Figure 10. The HCPT nanoparticles showed a more rapid dissolution than the unprocessed HCPT. After $120 \mathrm{~min}$, the dissolution rate of HCPT nanoparticles and unprocessed HCPT were $85.89 \%$ and $7.64 \%$, respectively. The HCPT nanoparticles had solubility eleven times higher than the unprocessed HCPT material. The dramatic increase in drug dissolution rate can be explained by the reduction of particle size resulting in an increased specific 
surface area. The literature has also discussed how an increase in the amorphous content and porosity of the micronized drugs led to an enhanced dissolution rate [26].

Figure 10. Dissolution profiles of the unprocessed HCPT and HCPT nanoparticles.

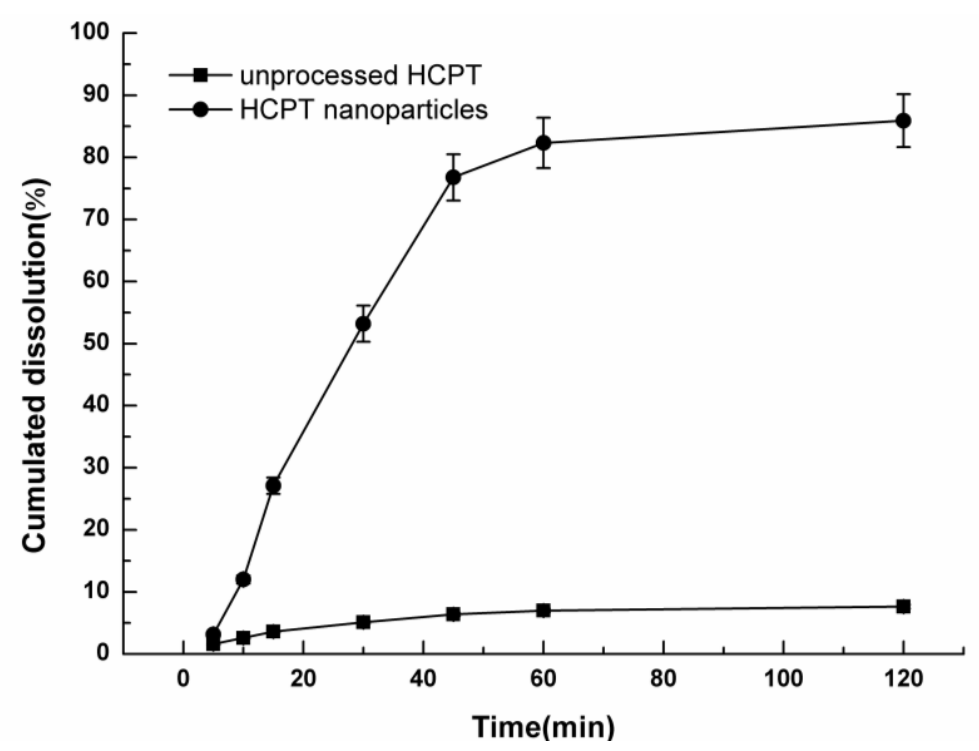

\section{Conclusions}

In the present work, a process for micronization of HCPT by supercritical antisolvent (SAS) has been developed. Of the four factors that have an effect on SAS micronization of HCPT from DMSO, three of the factors-concentration of HCPT solution, precipitation temperature and precipitation pressure-have a significant effect on the MPS of micronized HCPT, while the drug solution flow rate showed insignificant effects. The optimum micronization conditions are as follows: $35{ }^{\circ} \mathrm{C}$ of precipitation temperature, $20 \mathrm{MPa}$ of precipitation pressure, the flow rate ratio of $\mathrm{CO}_{2}$ and $\mathrm{HCPT}$ solution 19.55 and $0.5 \mathrm{mg} / \mathrm{mL}$ of HCPT solution concentration. Under the optimum conditions, HCPT nanoparticles with an MPS of $180 \pm 20.3 \mathrm{~nm}$ were obtained. The characterization data of the HCPT nanoparticles using SEM, DLS, FTIR, LC-MS, XRD and DSC showed on one hand, no degradation of HCPT is induced by the SAS process, on the other hand, the obtained HCPT particles have lower crystallinity. Additionally, there is a great increase of dissolution rate in HCPT nanoparticles by the SAS process compared to unprocessed HCPT. In conclusion, the SAS process proposed in the study proved an efficient method for the preparation of HCPT nanoparticles.

\section{Acknowledgements}

The authors are grateful for the precious comments and careful corrections made by anonymous reviewers. The authors would also like to acknowledge the financial support from the Special Fund for Forestry Scientific Research in the Public Interest (2010040072), 948 Project of the State Forestry Administration of China (2010-4-20) and the Fundamental Research Funds for the Central Universities (DL09BB08). The authors are also grateful to Lin Zhang for performing the LC-MS analysis. 


\section{References}

1. Yang, L.; Cui, F.; Cun, D.; Tao, A.; Shi, K.; Lin, W. Preparation, characterization and biodistribution of the lactone form of 10-hydroxycamptothecin (HCPT)-loaded bovine serum albumin (BSA) nanoparticles. Int. J. Pharm. 2007, 340, 163-172.

2. Wang, S.L.; Lin, S.Y.; Hsieh, T.F.; Chan, S.A. Thermal behavior and thermal decarboxylation of 10-hydroxycamptothecin in the solid state. J. Pharm. Biomed. Anal. 2007, 43, 457-463.

3. Hong, M.; Zhu, S.; Jiang, Y.; Tang, G.; Pei, Y. Efficient tumor targeting of hydroxycamptothecin loaded PEGylated niosomes modified with transferrin. J. Control. Release 2009, 133, 96-102.

4. Ma, J.; Liu, C.L.; Zhu, P.L.; Jia, Z.P.; Xu, L.T.; Wang, R. Simultaneous determination of the carboxylate and lactone forms of 10-hydroxycamptothecin in human serum by restricted-access media high-performance liquid chromatography. J. Chromatogr. B 2002, 772, 197-204.

5. Zhao, Y.X.; Hua, H.Y.; Chang, M.; Liu, W.J.; Zhao, Y.; Liu, H.M. Preparation and cytotoxic activity of hydroxycamptothecin nanosuspensions. Int. J. Pharm. 2010, 392, 64-71.

6. Takayama, H.; Watanabe, A.; Hosokawa, M.; Chiba, K.; Satoh, T.; Aimi, N. Synthesis of a new class of camptothecin derivatives, the long-chain fatty acid esters of 10-hydroxycamptothecin, as a potent prodrug candidate, and their in vitro metabolic conversion by carboxylesterases. Bioorg. Med. Chem. Lett. 1998, 8, 415-418.

7. Pessah, N.; Reznik, M.; Shamis, M.; Yantiri, F.; Xin, H.; Bowdish, K.; Shomron, N.; Ast, G.; Shabat, D. Bioactivation of carbamate-based 20(S)-camptothecin prodrugs. Bioorg. Med. Chem. 2004, 12, 1859-1866.

8. Ohwada, J.; Ozawa, S.; Kohchi, M.; Fukuda, H.; Murasaki, C.; Suda, H.; Murata, T.; Niizuma, S.; Tsukazaki, M.; Ori, K.; Yoshinari, K.; Itezono, Y.; Endo, M.; Ura, M.; Tanimura, H.; Miyazaki, Y.; Kawashima, A.; Nagao, S.; Namba, E.; Ogawa, K.; Kobayashi, K.; Okabe, H.; Umeda, I.; Shimma, N. Synthesis and biological activities of a pH-dependently activated water-soluble prodrug of a novel hexacyclic camptothecin analog. Bioorg. Med. Chem. Lett. 2009, 19, 2772-2776.

9. Angenault, S.; Thirot, S.; Schmidt, F.; Monneret, C.; Pfeiffer, B.; Renard, P. Cancer chemotherapy: A SN-38 (7-ethyl-10-hydroxycamptothecin) glucuronide prodrug for treatment by a PMT (Prodrug MonoTherapy) strategy. Bioorg. Med. Chem. Lett. 2003, 13, 947-950.

10. Zhang, L.; Yang, M.; Wang, Q.; Li, Y.; Guo, R.; Jiang, X.; Yang, C.; Liu, B. 10-Hydroxycamptothecin loaded nanoparticles: Preparation and antitumor activity in mice. J. Control. Release 2007, 119, 153-162.

11. Wang, A.; Li, S. Hydroxycamptothecin-loaded nanoparticles enhance target drug delivery and anticancer effect. BMC Biotechnol. 2008, 8, 46.

12. Shenderova, A.; Burke, T.G.; Schwendeman, S.P. Stabilization of 10-hydroxycamptothecin in poly(lactide-co-glycolide) microsphere delivery vehicles. Pharm. Res. 1997, 14, 1406-1414.

13. Mallery, S.R.; Shenderova, A.; Pei, P.; Begum, S.; Ciminieri, J.R.; Wilson, R.F.; Casto, B.C.; Schuller, D.E.; Morse, M.A. Effects of 10-hydroxycamptothecin, delivered from locally injectable poly(lactide-co-glycolide) microspheres, in a murine human oral squamous cell carcinoma regression model. Anticancer Res. 2001, 21, 1713-1722. 
14. Lu, B.; Zhang, Z. Novel colon-specific microspheres with highly dispersed hydroxycamptothecin cores: Their preparation, release behavior, and therapeutic efficiency against colonic cancer. J. Pharm. Sci. 2006, 95, 2619-2630.

15. Zhao, Y.; Gao, J.; Sun, X.; Chen, H.; Wu, L.; Liang, W. Enhanced nuclear delivery and cytotoxic activity of hydroxycamptothecin using o/w emulsions. J. Pharm. Pharm. Sci. 2007, 10, 61-70.

16. Zhang, C.; Ding, Y.; Yu, L.L.; Ping, Q. Polymeric micelle systems of hydroxycamptothecin based on amphiphilic $N$-alkyl- $N$-trimethyl chitosan derivatives. Colloid. Surface. B 2007, 55, 192-199.

17. Zhou, Y.Y.; Du, Y.Z.; Wang, L.; Yuan, H.; Zhou, J.P.; Hu, F.Q. Preparation and pharmacodynamics of stearic acid and poly (lactic-co-glycolic acid) grafted chitosan oligosaccharide micelles for 10-hydroxycamptothecin. Int. J. Pharm. 2010, 393, 144-152.

18. Pu, X.; Sun, J.; Wang, Y.; Liu, X.; Zhang, P.; Tang, X.; Pan, W.; Han, J.; He, Z. Development of a chemically stable 10-hydroxycamptothecin nanosuspensions. Int. J. Pharm. 2009, 379, 167-173.

19. Tavares Cardoso, M.A.; Geraldes, V.; Cabral, J.M.S.; Palavra, A.M.F. Characterization of minocycline powder micronized by a supercritical antisolvent (SAS) process. J. Supercrit. Fluid. 2008, 46, 71-76.

20. Kim, M.-S.; Jin, S.-J.; Kim, J.-S.; Park, H.J.; Song, H.-S.; Neubert, R.H.H.; Hwang, S.-J. Preparation, characterization and in vivo evaluation of amorphous atorvastatin calcium nanoparticles using supercritical antisolvent (SAS) process. Eur. J. Pharm. Biopharm. 2008, 69, 454-465.

21. Reverchon, E.; De Marco, I.; Torino, E. Nanoparticles production by supercritical antisolvent precipitation: A general interpretation. J. Supercrit. Fluid. 2007, 43, 126-138.

22. Chang, S.-C.; Lee, M.-J.; Lin, H.-M. Role of phase behavior in micronization of lysozyme via a supercritical anti-solvent process. Chem. Eng. J. 2008, 139, 416-425.

23. Dowy, S.; Braeuer, A.; Reinhold-López, K.; Leipertz, A. In situ optical monitoring of the solution concentration influence on supercritical particle precipitation. J. Supercrit. Fluid. 2010, 55, 282-291.

24. Reverchon, E.; Adami, R.; Cardea, S.; Porta, G.D. Supercritical fluids processing of polymers for pharmaceutical and medical applications. J. Supercrit. Fluid. 2009, 47, 484-492.

25. Zhao, X.; Zu, Y.; Li, Q.; Wang, M.; Zu, B.; Zhang, X.; Jiang, R.; Zu, C. Preparation and characterization of camptothecin powder micronized by a supercritical antisolvent (SAS) process. J. Supercrit. Fluid. 2010, 51, 412-419.

26. Chattopadhyay, P.; Gupta, R.B. Production of griseofulvin nanoparticles using supercritical CO(2) antisolvent with enhanced mass transfer. Int. J. Pharm. 2001, 228, 19-31.

(C) 2011 by the authors; licensee MDPI, Basel, Switzerland. This article is an open access article distributed under the terms and conditions of the Creative Commons Attribution license (http://creativecommons.org/licenses/by/3.0/). 